\title{
Aprendizaje y literacidad fuera de la escuela en la era digital: aproximaciones desde la ruralidad $^{1}$
}

\author{
Patricia Thibaut ${ }^{2}$ \\ ORCID: 0000-0003-2990-0339 \\ Margarita Calderón López ${ }^{3}$ \\ ORCID: 0000-0003-3805-5624
}

\section{Resumen}

En el marco de la era digital, el presente estudio explora las prácticas de literacidad de niños fuera de la sala de clases y su conexión con procesos de aprendizaje formal en un contexto rural. Utilizando una perspectiva ecológica y sociocultural, el artículo busca conectar áreas de investigación que frecuentemente se encuentran separadas en la literatura, como lo son los estudios centrados en la escuela y aquellos de aprendizaje informal; así como indagar en un área como es la educación rural que se encuentra subestudiada en la literatura. El estudio se sitúa en una localidad rural en Chile. Se utilizó una metodología de estudio de caso múltiple. Los resultados indican una orientación hacia las prácticas de literacidad digital en el hogar, pese a la falta de disponibilidad de tecnologías. Esto contrasta con la existencia de material impreso en los hogares y da cuenta de la importancia de distinguir conceptualmente el acceso y la disponibilidad hacia estos recursos (KALMAN, 2005). Se observa además que las prácticas que llevan a cabo los niños en su día a día son impulsadas por la entretención, la interacción con otros, el juego o el interés en aprender un tema específico. Por último, el análisis indica una discontinuidad con las prácticas de literacidad observadas en la sala de clases.

\section{Palabras clave}

Prácticas de literacidad - Aprendizaje - Educación rural - Nuevas tecnologías - Literacidad digital.

1- Este proyecto fue financiado por el Fondo Nacional de Desarrollo Científico de postdoctorado (Fondecyt) $n^{\circ} 3160114$, financiada por la Comisión Nacional de Investigación Científica y Tecnológica de Chile (Conicyt).

2- Universidad Austral de Chile, Valdivia, Chile. Contacto: patricia.thibaut@uach.cl.

3- Universidad de Chile, Chile. Contacto: margarita.calderon@uchile.cl. 


\section{Learning and out-of-school literacy practices in the digital age: a rural approach}

The present study explores children's out-of-school literacy practices and their connection with processes of formal learning in a rural context. The article aims at bridging research areas that are often disconnected in the literature, such as research on school education and informal learning; along with research on rural education that has been often underresearched in the literature. This research focuses the study of literacy and learning from an ecological and a socio-cultural perspective. The study is situated in a rural area in Chile and uses a multiple-case study design. The results show a trend towards digital literacy practices at home, despite the lack of availability of technologies. This contrasts with the presence of printed materials at home and bespeaks the need to distinguish between access to and availability of these resources (KALMAN, 2005). In addition, children's everyday practices are motivated by their interest on entertainment, interaction with others, play and the passion to learn a specific topic. Finally, the analysis shows a discontinuity between these practices and literacy practices observed in the classroom.

\section{Keywords}

Literacy practices - Learning - Rural education - New technologies - Digital literacies.

\section{Introducción}

Si bien desde hace décadas investigadores han iluminado procesos de aprendizaje fuera de la sala de clases, como por ejemplo desde un enfoque situado, de las comunidades de aprendizaje (LAVE et al., 1991; WENGER, 1998), desde la noción de mentoría (ROGOFF, 1990) o de aprendizaje distribuido (HUTCHINS, 1995), dichos enfoques se desarrollaron antes de la irrupción de la sociedad digital. Este factor es clave en tanto la sociedad de la información no sólo ha modificado las formas de intercambio, producción y comunicación de los adultos, sino que también ha impactado en la forma en que niños y adolescentes se comunican, aprenden y le dan sentido a su mundo (ITO et al., 2010). En general, la investigación en educación se ha centrado en la escuela (BARRON, 2006) y por lo tanto los resultados se encuentran desconectados de los procesos que se dan fuera de la sala de clases y de la mediación tecnológica en la que viven niños y adolescentes hoy en día (CABELLO; CLARO, 2017; FIELDS; GRIMES, 2012). Como plantea Barron (2006), cuando nos centramos sólo en la escuela para entender el aprendizaje, perdemos oportunidades para ver como el aprendizaje fluye desde sus propios actores y en el tiempo, espacio y las interacciones que naturalmente se dan en el día a día.

Respecto de la lectura y escritura, esta perspectiva adquiere gran relevancia en tanto que se trata de competencias básicas y fundamentales para desarrollar múltiples procesos de aprendizaje. La adquisición de estas habilidades implica un proceso largo y complejo que se desarrolla de manera fundamentalmente diferente a la adquisición del lenguaje 
oral ya que no ocurre de manera natural (ALEGRÍA, 1985). En este sentido, desde una perspectiva sociocultural, el aprendizaje de la lectura y la escritura ocurre en distintas instancias sociales y a través de prácticas e interacciones que ocurren entre personas (PAPEN, 2007). La lectura y escritura no solo se restringen a su adquisición; los nuevos estudios sobre la literacidad (CASSANY, 2005) comprenden la lectura y escritura como el desarrollo y proceso del lenguaje escrito en la sociedad y en eventos culturales reales (KALMAN, 2005).

El concepto prácticas de literacidad deriva del inglés literacy e intenta abarcar nuevas formas de creación y producción de significado que involucran prácticas canónicas como la lectura y escritura en papel, así como prácticas mediadas digitalmente como las que se realizan por ejemplo en un juego en línea, en una red social o en una plataforma de fanfiction (GEE, 2014). En el contexto comparado existe un creciente llamado para investigar y teorizar sobre dichas prácticas (ALVERMANN, 2008). Por otro lado, en el contexto chileno existe escasa evidencia respecto a los nexos entre tecnología, literacidad y aprendizaje que se dan dentro y fuera de la sala de clases.

Considerando esta evidencia, en la presente investigación adoptamos una perspectiva ecológica (BARRON, 2006) que busca iluminar las prácticas de literacidad fuera de la sala de clases, incluyendo los espacios, herramientas y actores donde éstas se desarrollan. En palabras de Barron, los jóvenes están “[...] simultáneamente inmersos en muchos lugares y son activos en crear contextos de actividad para ellos mismos dentro y entre lugares" (2006, p. 199). Cada contexto incluye arreglos específicos de "[...] actividades, recursos materiales, relaciones, y las interacciones que emergen desde ahí" (p. 196). Así, la perspectiva ecológica o conectada releva la interdependencia entre actores y contexto (IT0 et al., 2013) dando cuenta de manera explícita que el aprendizaje no es exclusivo de la escuela y que la relación con el entorno puede moldear los procesos de aprendizaje, tanto apoyando dicho aprendizaje como desincentivándolo (RAZETO, 2018).

Junto con la perspectiva ecológica, la teoría sociocultural guía la presente investigación y orienta la relación entre literacidad y aprendizaje. Desde este enfoque, ambos conceptos están íntimamente relacionados en tanto la comunicación entre quien aprende y un adulto o par con mayor conocimiento es vital para que se logre la zona de desarrollo próximo (VYGOTSKY, 1978). Esta mediación se estructura en el lenguaje. Además, la teoría sociocultural propone una segunda mediación basada en los artefactos disponibles para el aprendizaje. Así, se plantea que los artefactos elaborados por generaciones anteriores, como por ejemplo la escritura y otros sistemas de representación sirven como mediadores cognitivos y de prácticas sociales. Desde la perspectiva sociocultural, se entiende entonces que el aprendizaje depende (pero no está limitado) de las interacciones sociales, los artefactos disponibles y el momento histórico y cultural donde dichos procesos ocurren.

Por último, utilizaremos un enfoque de investigación centrado en los actores, en este caso los niños. Esta orientación es importante ya que permite explorar la realidad ya no desde la perspectiva del adulto, profesor o investigador, sino relevando a los actores como conocedores y constructores de sus propios procesos de aprendizaje y en consecuencia otorgando espacio a las voces de quienes han estado menos presentes en la investigación (CALDERON; THERIAULT, 2017). 
Las siguientes son las preguntas de investigación que guían este estudio: ¿Cuáles son las prácticas de literacidad de niños fuera de la sala de clases en contextos rurales? ¿Qué tipo de artefactos median dichas prácticas? ¿Cómo se relacionan dichas prácticas con el aprendizaje, la literacidad y la enseñanza formal de la escuela? Para contestar dichas preguntas, nos centraremos en un estudio de caso múltiple, realizado en una escuela rural multigrado, localizada en la región de los Ríos, Chile. En específico, analizaremos los casos de trece estudiantes de entre 10 y 11 años que atendian un curso multigrado de $5^{\circ}$ y $6^{\circ}$ básico.

\section{Aproximaciones teóricas al aprendizaje dentro y fuera de la sala de clases}

El aprendizaje en la escuela ha sido descrito con frecuencia como un aprendizaje basado en contenido, centrado en el profesor y donde el estudiante tiene un rol pasivo (KEMBER, 1997). Además se ha caracterizado por una noción de conocimiento que se transfiere y luego se posee (SFARD, 1998). Desde esta perspectiva, el conocimiento tiene un carácter estático en el que el aprendizaje generalmente se centra en el uso de la memoria y en una jerarquía profesor-alumno donde las interacciones en aula ocurren en una asimetría en la que el profesor posee el derecho a hablar y es quien guía las interacciones (CAZDEN, 1988). En esta formulación subyace una perspectiva epistemológica del aprendizaje en el que el contexto, los actores y los artefactos que se tienen a disposición son un telón de fondo para el desarrollo cognitivo y no un foco central. Además asume la resolución de problemas como un logro individual, más que como un trabajo cooperativo (SQUIRE, 2009). Como resultado, el aprendizaje en contextos formales en general tiene pocas conexiones con el entorno y con otros.

Por otro lado, el aprendizaje fuera de la sala de clases ha sido explorado desde hace décadas con el objetivo de dilucidar sus dimensiones analíticas (BROWN; COLLINS; DUGUIP, 1989; LAVE; WEGNER, 1991; WENGER, 1998). Así, la investigación fuera de la sala de clase ha examinado por ejemplo como individuos son capaces de resolver problemas matemáticos y lógicos utilizando las herramientas disponibles en el lugar (RESNICK, 1987). También ha mostrado la forma en que niños aprenden de manera informal y a través de prácticas situadas oficios complejos como el de sastrería (LAVE, 1988). Estos estudios han dado cuenta que existen diferencias en la resolución de problemas si comparamos un entorno formal de aprendizaje -como es la sala de clases- con un entorno fuera de la sala de clases. Plantean que mientras los problemas fuera de la sala de clases son contextualizados, prácticos y amplios, la escuela tienda a formular problemas descontextualizados, abstractos y limitados (RESNICK, 1987; SCRIBNER, 1984).

Uno de los cambios claves en el contexto del aprendizaje en el último tiempo es el de las nuevas tecnologías. Conceptos como el desarrollo de habilidades para el siglo XXI, o de las literacidades digitales, están en el discurso de la OECD, y de los programas educacionales en el contexto chileno (CHILE, 2015; OECD, 2014). No obstante, existe una desarticulación entre los usos de tecnología en la sala de clases y los usos que niños y adolescentes le dan fuera de ella. En Chile, por lo menos, las habilidades 
de Tecnología, Información y Comunicación se encuentran separadas de las habilidades cognitivas de orden superior y por lo tanto frecuentemente no se integran en la sala de clases (CHILE, 2013). Fuera de la sala de clases se observa en cambio, no sólo un aumento en el acceso, sino índices de uso de tecnología en niños y adolescentes similares a los de países desarrollados. Datos recientes indican, por ejemplo, que existe un amplio acceso a celulares inteligentes (smartphones) en todos los niveles socioeconómicos (92\%) y que un alto porcentaje de niños y adolescente (84\%) usan internet para actividades de aprendizaje tanto formal como informal. Además, la mayoría (91\%) de niños y adolescentes entre 9 y 17 años usuarios de internet ve videos o tutoriales en la web donde se enseña a hacer cosas que les gustan (CABELLO; CLARO, 2017).

Pese a ello, no es claro cómo se dan dichas prácticas y si tienen alguna conexión y relevancia en la trayectoria de los estudiantes para la educación formal o en su desarrollo. Los cambios observados a nivel de contexto tienen un correlato en el análisis de la literacidad, el cuál será abordado a continuación.

\section{Sobre el concepto de literacidad y sus (re)definiciones en la era digital}

El enfoque que desarrolla esta investigación emerge en conflicto con las perspectivas que abordan la lectura y escritura desde una perspectiva psicológica (GEE, 2014). Los nuevos estudios de literacidad o The new literacy studies cuestionan la lectura y escritura como procesos meramente cognitivos. Esta perspectiva teórica enfatiza la dimensión social que involucra la lectura y escritura. Este grupo de teoría define la lectura y escritura como un proceso social y cultural que responde a instituciones y propósitos diversos (BARRÉ-DE MINIAC; BRISSAUD, 2004; GEE, 1990; STREET, 1995). De acuerdo a Lankshear y Knobel (2003), existen dos tipos de alfabetización extraescolar: en primer lugar, las prácticas escolarizadas de lectura y escritura de los niños (PAHL; ROWSELL, 2005), que se refieren a todas las prácticas que los padres adoptan en el hogar para apoyar el desarrollo de la lectura y escritura de sus hijos y las prácticas que no tienen una base escolar: como las que incluyen juegos, lectura o escritura por placer, etc. Estas últimas surgen fuera del aula, en espacios comunitarios como la iglesia, el hogar, etc. y se relacionan con prácticas que surgen por iniciativa propia y que son placenteras para los estudiantes (BARTON, 1994; CAMITTA, 1993). Estas prácticas dan cuenta del contexto fuera de la escuela en el que están inmersos los estudiantes.

Las prácticas de literacidad fuera de la escuela (SPENCER; KNOBEL; LANKSHEAR, 2012) o prácticas de literacidad vernácula (BARTON; LEE, 2012; CAMITTA, 1993) involucran aquellas prácticas de lectura y escritura que no son reguladas por los procedimientos y reglas de instituciones sociales dominantes (como la escuela) y, por ende, tienen su origen en la vida cotidiana (BARTON; HAMILTON, 2012). Estas prácticas tienden a ser por iniciativa propia, es decir que son motivadas por un interés del estudiante para realizar la actividad. Las prácticas de literacidad vernáculas implican que las personas desarrollan estrategias informales para el aprendizaje, lo que significa todas las estrategias que las personas desarrollan fuera de los contextos formales de aprendizaje (BARTON; 
HAMILTON, 2012). En este sentido, la lectura y escritura vernácula se relacionan con estrategias particulares de aprendizaje y con sus estrategias de aprendizaje autogeneradas. Sin embargo, estas prácticas fuera de la escuela tienden a no ser reconocidas por el currículo de la educación básica (MARSH, 2006), aun cuando se vinculen a aprendizajes que los estudiantes desarrollan de manera voluntaria.

Estas prácticas vernáculas han adquirido gran relevancia en la vida diaria especialmente a través de los recursos y prácticas digitales. En la actualidad, niños y adolescentes realizan prácticas de lectura y escritura no sólo en papel y lápiz, sino que crecientemente en plataformas online. Las nuevas formas de participación en la vida social permiten la inclusión de las prácticas digitales de lectura y escritura entendidas como práctica social. Las prácticas digitales de lectura y escritura pueden ser definidas como aquellas prácticas que nos permiten rastrear significado a través de las tecnologías digitales (GILLEN; BARTON, 2010). Además, es relevante mencionar que los niños relacionan las prácticas digitales al juego (MARSH, 2010).

Por otro lado, las condiciones materiales asociadas a los participantes de este estudio explicitan la amplia gama de influencias que conforman su contexto. Estas condiciones forman parte de cómo se enfrenta y entiende el mundo y, por lo tanto, la literacidad. Las condiciones materiales con las que conviven los sujetos impactan en los recursos simbólicos y materiales para practicar la alfabetización. De acuerdo con Kalman (2005), este conjunto de relaciones, conocimientos y acciones constituyen las rutas de acceso y las modalidades de apropiación para desarrollar y usar la lectura y la escritura. El acceso contempla la disposición de los sujetos a acceder a recursos aun cuando no los tengan disponibles. Por otro lado, la disponibilidad abarca aquellos recursos que se encuentran disponibles y son familiares dentro del contexto de los participantes.

Esta distinción resulta de gran relevancia para este estudio puesto que el acceso que las personas tienen a recursos particulares y su disponibilidad proviene del contexto y del conjunto de experiencias de las personas involucradas y varía de acuerdo a cada participante, aun cuando estos compartan características socioculturales.

\section{Metodología}

El estudio contempla un diseño de caso múltiple holístico (YIN, 2009). Es decir, en el estudio cada niño es un caso en sí mismo y representa un corpus de datos respecto al tema de estudio. Con este enfoque se reforzó analíticamente la profundidad en el abordaje de las preguntas de investigación. En concreto, se exploraron las prácticas de literacidad en el entorno del hogar, así como sus comportamientos y percepciones.

\section{Contexto y participantes}

La investigación se sitúa en una pequeña localidad rural de la región de los Ríos en el sur de Chile. Su población es preferentemente campesina y se dedica a la agricultura, ganadería y actividad forestal. El establecimiento cuenta con una matrícula de alrededor de cuarenta alumnos, atendidos a nivel básico. De acuerdo a los datos del Sistema de Medición 
Escolar del Gobierno de Chile, la escuela se clasifica dentro del Grupo Socioeconómico Bajo (GSE). La mayoría de los padres y apoderados han declarado tener hasta 8 años de escolaridad y un ingreso del hogar hasta los \$300.000. Además, entre 72,01\% y 100\% de los estudiantes se encuentra en condición de vulnerabilidad social.

Los estudiantes tenían entre 10 y 11 años y cursaban 5 y 6 año básico en una misma sala de clases al momento de la recolección de datos. La tabla 1 indica las características de los participantes (todos los nombres en adelante han sido cambiados para cautelar el anonimato de sus participantes).

Tabla 1- Participantes del estudio

\begin{tabular}{|c|c|c|c|}
\hline Número & Pseudónimo & Edad & Curso \\
\hline 1 & Amaya & 11 & $6^{\circ}$ \\
\hline 2 & Bruno & 10 & $5^{\circ}$ \\
\hline 3 & Florencia & 10 & $5^{\circ}$ \\
\hline 4 & Paz & 11 & $6^{\circ}$ \\
\hline 5 & Ignacia & 11 & $6^{\circ}$ \\
\hline 6 & Jazmín & 11 & $6^{\circ}$ \\
\hline 7 & Pedro & 11 & $6^{\circ}$ \\
\hline 8 & Leonardo & 11 & $5^{\circ}$ \\
\hline 9 & Ricardo & 11 & $5^{\circ}$ \\
\hline 10 & Nieves & 11 & 6 \\
\hline 11 & Susana & 11 & $6^{\circ}$ \\
\hline 12 & Ana & 10 & $5^{\circ}$ \\
\hline 13 & Víctor & 10 & $5^{\circ}$ \\
\hline
\end{tabular}

Fuente. Elaboración propia.

\section{Recolección y análisis de datos}

La recolección de datos se llevó a cabo durante aproximadamente un semestre en el que se realizaron entrevistas semiestructuradas en las casas de los estudiantes. Dichas entrevistas estuvieron supervisados por sus padres o apoderados. En esta instancia, el foco se centró en explorar el acceso, disponibilidad, creencias y prácticas de literacidad y aprendizaje. El reporte hablado de los niños se complementó con las videograbaciones a sus prácticas en el entorno del hogar así como con las opiniones del apoderado del niño que se encontraba al momento de la entrevista. De este modo, las entrevistas lograron capturar los artefactos que los niños tenían a la mano como por ejemplo cuadernos, libros, tablets, así como sus prácticas como juegos o power points creados por ellos. Todas las entrevistas fueron grabadas en video y luego transcritas. Luego se realizó una segunda entrevista con los niños, esta vez en la escuela, para complementar la información obtenida en la primera instancia. Además, de manera de triangular los datos (MERRIAM; TISDELL, 2015), se incluyeron las observaciones de clases realizadas en la asignatura de Lenguaje durante un semestre, para lo cual se utilizó una pauta semiestructurada modificada de Milicic y otros autores (2008). 
El análisis de los datos de las entrevistas se realizó mediante un análisis temático con categorías predefinidas, a las que se sumaron una vez revisadas las transcripciones nuevas categorías. La codificación fue realizada por las dos investigadoras quienes revisaron de manera independiente las transcripciones y luego consensuaron las codificaciones en una revisión de pares. Los análisis se llevaron a cabo con el software Atlas Ti y en él se incorporaron los criterios de exhaustividad y exclusividad así como el de sensibilidad a las preguntas de investigación (MERRIAM; TISDELL, 2015). De este proceso se desprenden las interpretaciones y resultados que se discuten a continuación.

\section{Resultados}

\section{Recursos de literacidad: las vías de acceso y disponibilidad}

Respecto de los recursos de literacidad disponibles, se detectó una alta frecuencia de material impreso. De los trece estudiantes, ocho declaran tener materiales como revistas, historietas o libros. Estos materiales propician prácticas de literacidad en el hogar que se encuentran asociadas a la lectura en momentos de ocio. Sin embargo, los resultados sugieren que las prácticas cotidianas de literacidad están fuertemente vinculadas al uso de artefactos tecnológicos. La disponibilidad de este tipo de recursos para practicar la lectura y escritura de manera digital posee una alta presencia en los participantes en tanto que doce de los trece entrevistados menciona que utiliza celular, computador y/o tablet. Por otro lado, resulta relevante destacar que, pese a que solo tres estudiantes manifiestan tener acceso a internet en el hogar, esto no impide que accedan a este recurso a través de otros contextos familiares. Seis de los trece estudiantes acceden a internet aun cuando no disponen de internet en su hogar.

Las rutas de acceso de estos estudiantes incluyen la compra esporádica de bolsas para celulares (Paz), viajes a Valdivia (Ricardo) o el acceso a través de WiFi en lugares públicos (Paz y Jazmín). Sin embargo, y pese a que los participantes acceden a internet a través de rutas diversas, se manifiesta la falta de disponibilidad: la señal es mala y es un recurso costoso de adquirir en la zona. Sin embargo, estas carencias no impiden que todos los participantes declaren realizar alguna práctica de literacidad digital en el hogar. Ello da cuenta de que la falta de recursos para practicar la lectura y escritura digital no impide que estos estudiantes desarrollen un interés por utilizar y participar de la cultura digital.

A este respecto, cabe destacar la diversidad de aplicaciones que utilizan los estudiantes. De los trece estudiantes, siete declaran poseer Facebook y ocho utilizan WhatsApp. Por otro lado, respecto del uso que los estudiantes les dan a esas aplicaciones, Facebook destaca en cuanto a la frecuencia. Además, resulta relevante indicar que el uso que los estudiantes le dan a Facebook recae principalmente en la lectura, especialmente en la sección de noticias. Esta práctica de lectura digital involucra una decisión basada en sus intereses personales.

Por otro lado, WhatsApp posee un rol primordial en establecer comunicación con parientes o amigos cercanos. En este sentido, la escritura que realizan de manera digital se relaciona con los lazos afectivos y la mantención de vínculos fuera del entorno digital. 
Otra de las prácticas que adquiere gran relevancia en términos de periodicidad y relevancia son los juegos online. De los trece estudiantes, ocho declaran utilizar el computador y uno el celular para jugar en internet. En este sentido, se da cuenta de la importancia que adquiere el ocio dentro de las prácticas digitales de lectura y escritura.

\section{Prácticas de literacidad: la lectura y escritura en el día a día}

Las actividades que realizan los estudiantes diariamente son variadas y cubren un espectro en el que mencionan desde juntar leña hasta jugar videojuegos online como actividades que realizan en el hogar. Esta variedad de actividades posee objetivos y características diversos y no todas involucran la lectura y escritura. Sin embargo, en este artículo nos referiremos a aquellas actividades fuera de la escuela que involucran a la literacidad. En relación con las prácticas escolarizadas (PAHL; ROWSELL, 2005), cabe destacar que típicamente involucran el tiempo en el hogar que los estudiantes dedican a las tareas escolares. De este proceso se desprenden los resultados que se discuten a continuación. Se incluyen en los resultados una serie de citas extraídas de las entrevistas realizadas con los estudiantes de manera de ilustrar los análisis.

Por ejemplo, Amaya, Jazmín, Pedro, Ricardo, Susana, Víctor y Florencia mencionan el tiempo dedicado a las tareas o el estudio como una actividad propia de la rutina diaria. Sin embargo, resulta relevante mencionar que los estudiantes describen esta actividad como una parte importante de sus actividades relacionadas a la lectura y escritura en el hogar. Por ejemplo, cuando la entrevistadora pregunta a Jazmín si escribe en la casa, Jazmín menciona: “No, acá. En la casa no escribo. Y cuando tengo tareas no más”. Su comentario releva la escritura solo a las tareas. Ricardo también declara que lee solo "cuando tengo que hacer tarea”. Lo mismo sucede con Pedro, Susana y Víctor.

Sin embargo, resulta relevante indicar que Pedro, Susana y Víctor declaran desarrollar una amplia gama de prácticas digitales en las que utilizan la lectura y escritura. En este sentido, estos participantes no relacionan estas prácticas digitales a la lectura o escritura propiamente tal.

Por otro lado, cuando se indagó en las prácticas digitales, once de los trece estudiantes declararon desarrollar diversas actividades asociadas a los recursos disponibles. Estos resultados dan cuenta de cómo las prácticas digitales no fueron mencionadas cuando la entrevistadora preguntó por la lectura y escritura, lo que nos permite inferir que los estudiantes no necesariamente reconocen la lectura y escritura como las habilidades que motivan su actividad digital.

En este sentido, estas prácticas digitales se relacionan a prácticas de literacidad vernácula en tanto que estas no son motivadas por la intención de leer o escribir, sino que la lectura y escritura se desarrollan como un aspecto secundario de la actividad (MARSH, 2014). Algo similar ocurre con Amaya, quien cuando es preguntada por la entrevistadora si le gusta leer y escribir, declara que “más o menos'. No obstante, once de los trece estudiantes realizan variadas prácticas fuera de la sala de clases por iniciativa propia. Por ejemplo, se menciona escribir un cuaderno de canciones (dos estudiantes), escribir cuentos (3 estudiantes) o realizar lectura o escritura en plataformas digitales (once estudiantes). Por ejemplo, al nombrar una de sus prácticas señala: 
A: Este es un cuaderno que hice. Escribo canciones ...esas son para mis compañeros, esas son las que les gustan.

E: ¿Ah.. y tú pasas en limpio las canciones? ¿Las escuchas y las escribes?

A: Sí

E ¿Y a dónde las escuchas?

A: En el mp3

E: ¿Y cómo las bajas?

A: Me las pasa mi hermana. Es que donde hace su práctica ella tiene internet entonces le aprovecha de descargar música. (Amaya).

Este y otros comentarios dan cuenta que la entretención es uno de los motivadores de las prácticas vernáculas en el día a día. Por ejemplo, en relación con la lectura en Facebook, Paz describe como sus prácticas de lectura en Facebook se orientan por sus intereses:

E: ¿Y el Facebook, de lo que has ocupado, que has leído ahí, o que haces?

P: No, es que de repente como que me aparecen, no sé, "15 imágenes que te darán mucha risa", no sé, y ahí empiezo a leer; o por ejemplo, de repente me aparecen historias de hace muchos años atrás, o de repente cosas que, no sé, son muy raras, como por ejemplo de un hombre que murió en el año 37, y ese mismo año nació otro hombre y es igual.

E: Como datos curiosos

P: Sí. (Paz).

Además, se observa que las herramientas de la cultura popular con que los niños se entretienen son variadas y van desde lo análogo a lo digital, incluyendo cómics como Condorito $^{4}$, libros, revistas, memes, canciones que descargan de YouTube, películas o videojuegos. Al respecto, Ito y otros autores (2010) señala que los niños y jóvenes utilizan el contenido de la cultura popular como una forma de elaborar y distinguir su identidad y para negociar la relación con sus pares. Por ejemplo, en el comentario siguiente vemos como Paz utiliza distintos formatos, herramientas y modos, en los cuales se combinan prácticas de escritura tradicional con prácticas digitales.

E: ¿Y acá de lo que buscas es más para entretenerte?

$\mathrm{P}:$ He, de repente cuando miro tele es para escuchar música, y mi mamá varias veces me ha retado porque pongo la música muy alta pero cuando hay canciones que no sé anoto los nombres y después en Google busco la letra de la canción y la escribo y después de haberla escrito la busco aquí para verla y la escucho, la escucho la escucho hasta que me aburro, pero cuando me aburro me la aprendo. (Paz).

Las prácticas de literacidad motivadas por la comunicación con pares y amigos se destacan dentro de las actividades realizadas por los estudiantes fuera de la sala de clases en tanto el tiempo y la frecuencia destinado a este propósito. Se observa que los niños usan las redes sociales para continuar conectados a sus compañeros de clase o

4- Condorito es una historieta cómica chilena que ha alcanzado popularidad en toda Latinoamérica. 
mantener comunicación con familiares o amigos que viven lejos. Esto coincide con los datos reportados en la encuesta Kidsonline ${ }^{5}$ y también a nivel internacional (ROTHONI, 2017). Esta evidencia sugiere además que más que generar vínculos con desconocidos lo que se busca es extender la comunicación de conocidos fuera de las instancias cara a cara.

Además, en muchos casos los niños generan estrategias y alianzas con sus padres y madres para poder participar en este tipo de actividades. Como lo relata la madre de Paz, "[...] supuestamente el Facebook lo puede crear una persona mayor de edad y en este caso las dos lo creamos, pero le cambiamos la fecha. Sí, porque no se puede”. Cuando esto no sucede algunos niños elaboran formas de obviar dichas limitaciones para poder participar de este tipo de prácticas sociales. "La verdad es que se creó un Facebook escondido de nosotros y como no nos pidió permiso le dijimos que tenía que cerrarlo porque en redes sociales uno no sabe quién está detrás de esa pantalla y ella es muy chiquitita” (Madre de Amaya).

Las prácticas de literacidad motivadas por el juego ocupan un lugar relevante dentro de las actividades de los participantes y surgen a partir del gozo que experimentan. Por ejemplo, Bruno también menciona que le gusta jugar juegos online y que para ello debe leer:

E: ¿Y para jugar tienes que leer?

B: No, bueno primero te dan las indicaciones y ahí uno tiene que leer. Hay un juego que empezaba y eran como dos páginas de cuaderno; no como 5 páginas de cuaderno y yo empecé a leer, a leer y como a la $3^{\circ}$ me aburrí y me empecé a adelantar. (Bruno).

En ese caso, Bruno ejemplifica como el interés por el juego propicia la lectura. Sin embargo, debido a que la lectura no es la actividad que lo motiva, el estudiante pierde la motivación cuando la lectura ocupa la mayor parte de la actividad.

En este sentido, en este estudio distinguimos aquellas prácticas motivadas por el juego y el entretenimiento de aquellas motivadas por el aprendizaje sobre un tema que apasiona al niño, las que serán abordadas en el siguiente acápite.

\section{Prácticas motivadas por el aprendizaje de un tema específico: el caso de Pedro}

El análisis de los datos da cuenta de que existen ciertas prácticas en la que los niños manifiestan un interés explícito en aprender. Este tipo de prácticas se puede vincular con conceptualizaciones elaboradas bajo nombres distintos y que provienen de corrientes diversas como la antropología cultural o la lingüística social. Por ejemplo, Ito y otros autores (2013) definen este tipo prácticas como aprendizaje conectado, el cual se caracteriza por estar basado en el interés de los niños; por incluir una fuerte participación y apoyo de pares; y por tener una orientación hacia el conocimiento del área escogida. Este tipo de prácticas se orientan a un tema específico de interés del niño o adolescente. Además, se diferencian de prácticas de consumo pasivo y de entretenimiento -como ver películas o ver videos en internet- en tanto el niño o adolescente muestra un interés

5- Kids online es una red internacional de colaboración que incluye a 33 países. Su objetivo es coordinar y estimular investigación sobre la forma en la que niños usan nuevos medios, incluyendo los riesgos y la seguridad online. 
activo en aprender. Por otro lado, Gee elabora la noción de espacios de afinidad en la que "[...] novicios, maestros y todo el mundo trabajan juntos y socializan en torno a una tarea en común" (2005, p. 85). Estas aproximaciones tienen en común el hecho de que el aprendizaje se construye con distintos niveles de conocimiento sobre un tema, en espacios virtuales y cara a cara, y en los cuales la formación de redes es fundamental.

El caso de Pedro es un ejemplo que se orienta a un aprendizaje conectado, en el cual el niño desarrolla un interés y conocimiento sobre las bicicletas que inicia fuera de la sala de clases. Pedro señala que le gusta ir a la escuela y al preguntarle cómo se describiría como alumno, indica que "más o menos" porque no se considera en sus palabras "muy santito", "muy ordenado". Le gusta estudiar, pero menciona que no le gusta leer ni escribir porque lo aburre. No obstante, su respuesta cambia cuando distingue el tipo de lectura. "0 sea sí, pero depende si me gusta lo que estoy leyendo o no”. En una segunda entrevista, Pedro vuelve a reportar las mismas creencias sobre la lectura. En sus palabras:
E: ¿Qué te gusta leer?
P: No sé, es que depende de la... de qué se trata o no.
$\mathrm{E}:$ ¿Qué sería interesante de leer?
P: De bicicletas. (Pedro).

Esta es una respuesta frecuente en la investigación. La literatura reporta que niños y niñas, pero en especial los niños (hombres), son muy dependientes de si les gusta lo que están leyendo (STEINKUEHLER; COMPTON-LILLY; KING, 2010). En escritura la investigación en Chile muestra resultados similares, relevando la importancia de la motivación en la infancia (GÓMEZ et al., 2016).

La tipología de Barron (2006) es útil para ilustrar los procesos de construcción de conocimiento que se desprenden de prácticas orientadas a un aprendizaje conectado, las cuales se detallen en la Cuadro 1.

Cuadro 1- Tipos de estrategias de autoiniciadas de construcción de conocimiento, adaptado de Barron (2006)

\begin{tabular}{|l|l|}
\hline \multicolumn{1}{|c|}{ Tipo } & \multicolumn{1}{c|}{ Ejemplo de estrategias } \\
\hline Encontrar información basada en textos & Identifica y lee libros, revistas o información de internet \\
\hline $\begin{array}{l}\text { Crear actividades interactivas } \\
\text { Explorar medios }\end{array}$ & $\begin{array}{l}\text { Completa tutoriales, crea nuevos proyectos, empieza un negocio } \\
\text { Experimenta con programas, explora en la web, analiza las creaciones de otros }\end{array}$ \\
\hline $\begin{array}{l}\text { Buscar aprendizaje estructurado } \\
\text { Construir redes de conocimiento }\end{array}$ & $\begin{array}{l}\text { Se inscribe en cursos dentro o fuera de la escuela, se une a un club } \\
\text { Se une a grupos de interés, encuentra mentores, tiene conversaciones con pares } \\
\text { o parientes }\end{array}$ \\
\hline
\end{tabular}

Fuente: Elaboración propia.

Varios participantes realizan diversas estrategias autoiniciadas de construcción de conocimiento. Sobre todo, en lo que respecta a la lectura y búsqueda de información, así como a la creación de proyectos para lo cual utilizan redes sociales, YouTube o 
la Web. De manera similar, Pedro busca y encuentra información relacionada con las bicicletas. No obstante, el uso de la tecnología, a diferencia de los casos vistos más arriba, tiene un objetivo orientado a su pasión y por lo tanto se observan menores funciones asociadas a interactuar con amigos o mirar sin un objetivo claro. También, a través de Instagram y Facebook se mantiene informado de los eventos sobre bicicletas y las últimas actualizaciones. Además, a través de esta vía es parte de una red de pares interesados en este deporte. Por ejemplo, al preguntarle sobre sus prácticas digitales Pedro indica:
E: ¿Qué haces en Facebook?
P: Veo videos.
E: ¿Y lees noticias, de esas que de repente salen en el Inicio?
P: Sí, de repente. Si me interesan también.
E: ¿Qué te interesa leer en Facebook?
P: De bicis. De quién ganó la copa mundial, por decirlo así. (Pedro).

Lo mismo sucede con los juegos en línea que practica. Su interés guía la selección de juegos que realiza:

E: ¿Si tuvieras que mostrarme un juego de tu celular?

P: Este es de bici (risas). (Pedro).

También es interesante destacar que Pedro conjuga prácticas de literacidad en espacios digitales con la práctica de su hobby en los cerros aledaños a su casa. Cuando la entrevistadora le pregunta qué hace cuando llega de la escuela Pedro señala "Si es que no tengo tareas, salgo. Al cerro, a bajar en bici”.

Figura 1- Participación en eventos de literacidad relacionado con las bicicletas

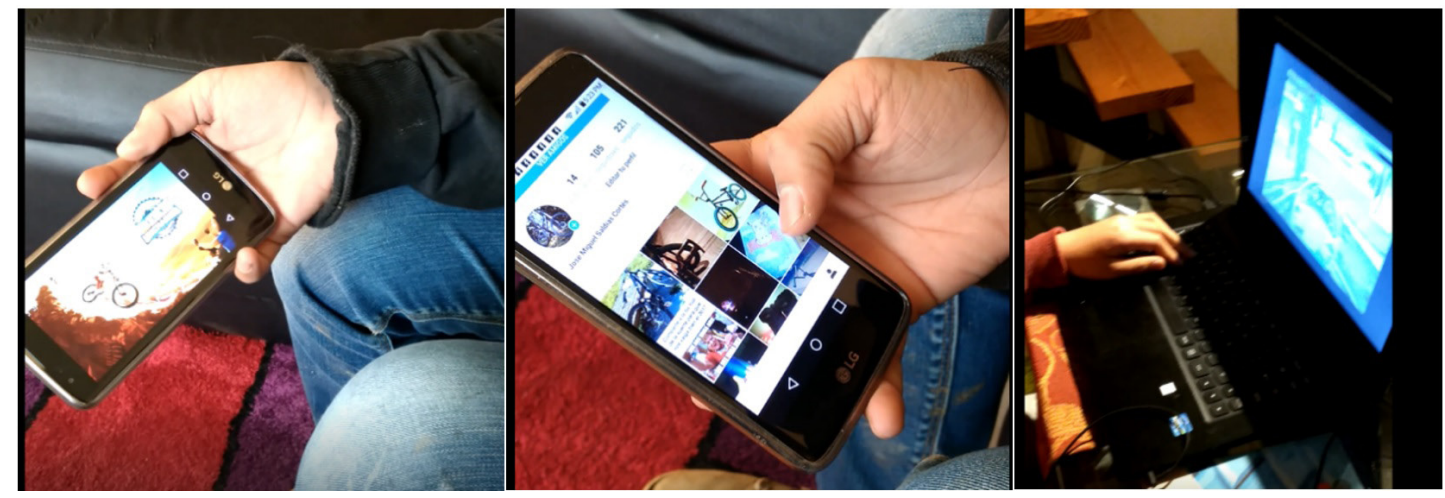

Fuente: Pedro. Prácticas digitales de Pedro. Elaboración propia.

Pedro, de manera consistente, da cuenta que utiliza los recursos disponibles con el foco de aprender y estar al día en el área de su interés: las bicicletas. Si utilizamos el concepto de ecología y contexto de Barron (2006), podemos dar cuenta que Pedro es un 
niño activo que está creando sus propios contextos de actividades. Resulta interesante ver como estas actividades sobrepasan la disponibilidad de recursos materiales, así como lo aprendido en la escuela. Éste último punto pudo constatarse a la luz de las observaciones realizadas durante un semestre en la sala de clases a la que asistían los participantes del estudio, las cuales indicaron un muy bajo uso de tecnologías en la asignatura de Lenguaje.

\section{Discusión e implicancias}

Al comienzo del artículo planteamos la necesidad de explorar las prácticas de literacidad fuera de la sala de clases y su relación con procesos de aprendizaje. Para ello pusimos especial atención en identificar los artefactos que los niños que estudian en un contexto rural tienen a la mano para leer y escribir, así como en los haceres vinculados a dichas herramientas. Esto es relevante en tanto la forma de ver y enfrentar el mundo es moldeada de manera importante por las condiciones materiales. Al respecto, el estudio muestra que los participantes disponen de material impreso para el fomento de prácticas de literacidad como libros, cuentos y cuadernos lo cual contrasta con la mirada general que existe a nivel país sobre la baja cantidad de recursos materiales para la lectura y la escritura en la casa (CALDERÓN LÓPEZ, 2017; STRASSER; LISSI, 2009).

No obstante, las prácticas más frecuentes realizadas fuera de la sala de clases se orientan a la literacidad digital. Esto es interesante ya que, pese a que se evidencia una falta de disponibilidad material de artefactos tecnológicos como WiFi o computador personal, los niños elaboran estrategias para acceder y en consecuencia participar en prácticas de literacidad que son propias de la cultura digital. Éstas incluyen desde copiar canciones en el computador, conversar con amigos y parientes por redes sociales, jugar en línea, hasta participar en espacios de afinidad y eventualmente en comunidades de práctica para especializarse en un tema como las bicicletas. En este último caso, los resultados indican que incluso a una temprana edad, algunos estudiantes como Pedro encuentran sus propias estrategias para aprender y participar en comunidades de su interés. En este respecto, Gee (2014) plantea que los espacios de afinidad son lugares donde se puede desarrollar el aprendizaje informal. Estos espacios físicos, digitales o combinados pueden ocurrir a lo largo de distintas entradas o portales como blogs, páginas web o encuentros cara a cara (LAMMERS; CURWOOD; MAGNIFICO, 2012).

Por otro lado, los resultados del estudio revelan una fusión entre la cultura escrita y la digital en la que los participantes transitan naturalmente entre distintos espacios, textos y formas de rastrear significado y hacer sentido de su mundo. Así, la continuidad entre el espacio análogo y el digital es uno que no resulta problemático para los niños y que se orienta a la realización de algo, sea esto escribir un cuento, documentar un paseo o conocer más sobre las bicicletas.

En resumen, los datos analizados indican que existe una variedad de prácticas de literacidad desarrolladas por los niños que viven en áreas rurales, así como en las estrategias, espacios y herramientas que los niños usan fuera de la sala de clases. Pese a ello se observa que las prácticas digitales no son percibidas por ellos como prácticas 
de literacidad. Éstas últimas se asocian con hacer tareas y con actividades que no son motivantes.

A nivel de implicancias de los resultados, las percepciones de los niños sobre lo que identifican como literacidad es una señal de alerta con respecto a la forma en la que se conceptualiza y se generan prácticas de literacidad en la escuela. La evidencia del estudio muestra que la motivación es un factor promotor de las prácticas de literacidad fuera del aula. En consecuencia, el estudio sugiere que una de las formas de generar puentes entre el aprendizaje formal y el aprendizaje en el día a día es atender de manera explícita a las motivaciones que los niños tienen fuera de la escuela y fomentar el desarrollo de estos intereses en el aula. Esto también se puede vincular con el carácter social que evidencian las prácticas de literacidad observadas en la muestra (LANKSHEAR; KNOBEL, 201). Así, la observación fuera del aula indica que las prácticas de literacidad realizadas por los niños se desarrollan principalmente en las siguientes instancias: por iniciativa propia y motivadas por el interés sobre un tema o por entretenimiento; cuando hay interacción entre los niños; o cuando se vincula con el juego. Por lo tanto, estos aspectos no debiesen estar ajenos a la enseñanza-aprendizaje en el aula.

Desde la perspectiva del aprendizaje fuera de la sala de clases, vemos que un aspecto importante a relevar en la escuela es el aprendizaje de pares. En particular, el caso de Pedro da cuenta de oportunidades de aprendizaje que se generan al interactuar con pares con trayectorias diversas cuyo punto de encuentro es accesible desde distintos portales como Instagram, Facebook o el cerro cercano a la casa de Pedro. Al respecto, Lave y Wegner (1991) plantean que a través de este tipo de participación los niños podrían eventualmente ser parte de una comunidad de práctica y construir una identidad propia que entrega posibilidades de aprendizaje distintas a las consideradas sólo en el marco del aprendizaje formal. Fomentar la interacción con otros y destacar los conocimientos que cada niño trae es algo que no es suficientemente fomentado en el aprendizaje de aula y, como lo indica la investigación, la tecnología puede abrir espacios que promuevan el aprendizaje social (THIBAUT et al., 2015).

Por último, la alta frecuencia de prácticas digitales contrasta con la forma en la que en general se discute y enseña literacidad en espacios formales como la escuela, en los que los usos tecnológicos se separan de los contenidos y se otorga estatus distintos a las prácticas canónicas de las digitalmente mediadas (DAVIDSON, 2009). Como consecuencia, la práctica de escritura y lectura escolar es muchas veces insular y desarticulada de situaciones y herramientas auténticas de aprendizaje. Al respecto Curwood y Cowell plantean que

[...] en un mundo donde la comunicación sincrónica y asincrónica es fácilmente disponible, las herramientas digitales son omnipresentes, y en la que los espacios online entregan infinitas posibilidades, es crítico que los educadores entiendan cómo las prácticas de literacidad operan en dichos contextos. (2011, p. 110).

Finalmente, y dado el carácter cualitativo del estudio, es importante en futuras investigaciones ahondar en el alcance de esta investigación a otras localidades rurales 
y urbanas del país. Además, la investigación sugiere como líneas de acción a seguir la identificación de estrategias efectivas para fomentar el nexo entre escuela y contexto atendiendo a las especificidades de los niños y a las herramientas a las que se puede acceder en el aula para expandir los procesos de aprendizaje vinculados a la literacidad. El rol del docente, pero también las estrategias a nivel escuela para apoyar estos vínculos son fundamentales para lograr dichas redes. Tal como evidencia la investigación comparada, los conocimientos, habilidades y recursos que poseen las familias y en específico los niños no son triviales en los procesos de aprendizaje y por lo tanto es vital generar puentes que conecten las prácticas dentro y fuera de la sala de clases (MOLL et al., 1992). Esto es particularmente importante en familias que viven en contextos rurales, en tanto se puede potenciar el capital social y cultural en zonas que por lo general están en condiciones de aislamiento con respecto a núcleos de desarrollo tradicional.

\section{Referencias}

ALEGRÍA, Jesús. Por un enfoque psicolingüístico del aprendizaje de la lectura y sus dificultades. Infancia y Aprendizaje, Madrid, n. 29, p. 79- 94, 1985.

ALVERMANN, Donna. Why bother theorizing adolescents' online literacies for classroom practice and research? Journal of Adolescent \& Adult Literacy, Newark-Hoboken, v. 52, n. 1, p. 257-274, 2008.

BARRÉ-DE MINIAC, Christine; BRISSAUD, Catherine. La littéracie: conceptions théoriques et pratiques d'enseignement de la lecture-écriture. París: L'Harmattan, 2004.

BARRON, Brigid. Interest and self-sustained learning as catalysts of development: a learning ecology perspective. Human Development, Davis, v. 49, n. 4, p. 193-224, 2006.

BARTON, David. An introduction to the ecology of written language. Hoboken: Wiley, 1994.

BARTON, David; HAMILTON, Mary. Local literacies: reading and writing in one community. London: Routledge. 2012.

BARTON, David; LEE, Carmen. Redefining vernacular literacies in the age of Web 2.0. Applied Linguistics, Oxford, v. 33, n. 3, p. 282-298, 2012.

BROWN, John Seely; COLLINS, Allan; DUGUID, Paul. Situated cognition and the culture of learning. Educational Researcher, Champaign, v. 18, n. 1, p. 32-42, 1989.

CABELLO, Patricio; CLARO, Magdalena. General results, kids online survey Chile. Santiago de Chile: Kids Online Chile, 2017.

CALDERÓN LÓPEZ, Margarita. Self-generated literacy practices in disadvantaged environments in Chile. International Journal of Education and Literacy Studies, Doncaster, v. 5, n. 1, p. 29-41, 2017. 
CALDERON LOPEZ, Margarita; THERIAULT, Virginie. Accessing a 'very, very secret garden': exploring the literary practices of children and young people using participatory research methods. Language and Literacy, Alberta, v. 19, n. 4, p. 39-54, 2017.

CAMITTA, Miriam. Vernacular writings: varieties of literacy among Philadelphia high school students. Crosscultural approaches to literacy. Cambridge: Cambridge University Press, 1993.

CASSANY, Daniel. Investigaciones y propuestas sobre literacidad actual: multiliteracidad. In: CONFERENCIA INAUGURAL DEL CONGRESO NACIONAL CÁTEDRA UNESCO PARA LA LECTURA Y LA ESCRITURA, 2005, Concepción. Conferencia... Concepción: Unesco, 2005. p. 1-10.

CAZDEN, Courtney. Classroom discourse: the language of teaching and learning. Portsmouth: Heinemann, 1988.

CHILE. Comité de Ministros para el Desarrollo Digital. Agenda digital 2020: Chile digital para todos. Santiago de Chile: [s. n.], 2015.

CHILE. Ministerio de Educación. Desarrollo de habilidades digitales para el siglo XXI en Chile: ¿Qué dice el SIMCE TIC? Santiago de Chile: [s. n.], 2013.

CURW00D, Jen Scott; COWELL, Lora Lee. iPoetry: creating space for new literacies in the English curriculum. Journal of Adolescent \& Adult Literacy, Newark-Hoboken, v. 55, n. 2, p. 110-120, 0ct. 2011.

DAVIDSON, Christina. Young children's engagement with digital texts and literacies in the home: pressing matters for the teaching of English in the early years of schooling. English Teaching, Bingley, v. 8, n. 3, p. 36-54, 2009.

FIELDS, Deborah; GRIMES, Sara. Kids online: a new research agenda for understanding social networking forums. Irvine: The Joan Ganz Cooney Center, 2012.

GEE, James Paul. Multimodal social semiotics: writing in online contexts. In: ROWSELL, Jennifer; PAHL, Kate (Ed.). The routledge handbook of contemporary literary studies. London: Routledge, 2014. p. 1-19.

GEE, James Paul. Semiotic social spaces and affinity spaces. In: BARTON, David; TUSTING, Karin (Ed.). Beyond communities of practice: language power and social context. Massachusetts: Cambridge University Press, 2005. p. 214-232.

GEE, James Paul. Social linguistics and literacies: ideology in discourses. Thousand Oaks: Routledge, 1990.

GILLEN, Julia; BARTON, David. Digital literacies: a research briefing by the technology enhanced learning phase of the teaching and learning research programme. London: TLRP, 2010. Technology Enhanced Learning Programme.

GÓMEZ, Gabriela et al. La producción escrita de estudiantes y escuela en base a resultados SIMCE, factores contextuales y modelos de buenas prácticas docentes. Santiago de Chile: Mineduc, 2016. 
HUTCHINS, Edwin. Cognition in the wild. Cambridge: MIT Press, 1995.

ITO, Mizuko et al. Hanging out, messing around, geeking out: living and learning with new media. Cambridge: MIT Press, 2010.

ITO, Mizuko et al. Connected learning: an agenda for research and design. California: Digital Media and Learning Research Hub, 2013.

KALMAN, Judy. Access routes to written culture for a group of women in Mexico. [S. I.]: Unesco, 2005.

KEMBER, David. A reconceptualisation of the research into university academics' conceptions of teaching. Learning and Instruction, London, v. 7, n. 3, p. 255-275, 1997.

LAMMERS, Jayne; CURWO0D, Jen Scott; MAGNIFICO, Alecia Marie. Toward an affinity space methodology: considerations for literacy research. English Teaching, New Zealand, v. 11, n. 2, p. 44-58, 2012.

LANKSHEAR, Colin; KNOBEL, Michele. New literacies: everyday practices and social learning. New York: Open University Press, 2011.

LANKSHEAR, Colin; KNOBEL, Michele. New technologies in early childhood literacy research: a review of research. Journal of Early Childhood Literacy, California, v. 3, n. 1, p. 59-82, 2003.

LAVE, Jean. Cognition in practice: mind, mathematics, and culture in everyday life. Cambridge: Cambridge University Press, 1988.

LAVE, Jean; WENGER, Etienne. Situated learning: legitimate peripheral participation. Massachusetts: Cambridge University Press, 1991.

LLOPART, Mariona; ESTEBAN-GUITART, Moisès. Funds of knowledge in 21st century societies: inclusive educational practices for under-represented students: a literature review. Journal of Curriculum Studies, United Kingdom, v. 50, n. 2, p. 145-161, 2018.

MARSH, Jackie. Popular culture in the literacy curriculum: a bourdieuan analysis. Reading Research Quarterly, Hoboken, v. 41, n. 2, p. 160-174, 2006.

MARSH, Jackie. Purposes for literacy in children's use of the online virtual world Club Penguin. Journal of Research in Reading, Hoboken, v. 37, n. 2, p. 179-195, 2014.

MARSH, Jackie. Young children's play in online virtual worlds. Journal of Early Childhood Research, Newbury Park, v. 8, n. 1, p. 23-39, 2010.

MERRIAM, Sharan B.; TISDELL, Elizabeth J. Qualitative research: a guide to design and implementation. San Francisco: Jossey-Bass, 2015.

MILICIC, Neva et al. Diseño, construcción y evaluación de una pauta de observación de videos para evaluar calidad del desempeño docente. Psykhe, Santiago de Chile, v. 17, n. 2, p. 79-90, 2008. 
MOLL, Luis C. et al. Funds of knowledge for teaching: using a qualitative approach to connect homes and classrooms. Theory Into Practice, Oxford, v. 31, n. 2, p. 132-142, 1992.

OECD. Organización para la Cooperación y Desarrollo Económicos. Education at a glance 2014: OECD Indicators. Paris: OECD, 2014.

PAHL, Kate; ROWSELL, Jennifer. Literacy and education: understanding the new literacy studies in the classroom. London: Paul Chapman, 2005.

PAPEN, Uta. Literacy and globalization: reading and writing in times of social and cultural change. London: Routledge, 2007.

RAZETO, Alicia. Estrategias para promover la participación de familias en la educación de niños en escuelas chilenas. Educação e Pesquisa, São Paulo, v. 44, e180495, p. 1-20, 2018.

RESNICK, Lauren B. Learning in school and out. Educational Researcher, California, v. 16, n. 9, p. 13-20, 1987.

ROGOFF, Barbara. Apprenticeship in thinking: cognitive development in social context. New York: Oxford University Press, 1990.

ROTHONI, Anastasia. The interplay of global forms of pop culture and media in teenagers' 'interest-driven' everyday literacy practices with English in Greece. Linguistics and Education, Amsterdan, v. 38, p. 92-103, Apr. 2017.

SCRIBNER, Silvya. Studying working intelligence. In: ROGOFF, Barbara; LAVE, Jean (Org.). Everyday cognition: Its development in social context. Cambridge: Cambridge University Pres, 1984. p. 9-40.

SFARD, Anna. On two metaphors for learning and the dangers of choosing just one. Educational Researcher, California, v. 27, n. 2, p. 4-13, 1998.

SPENCER, Tamara; KNOBEL, Michele; LANKSHEAR, Colin. Researching young children's out-of-school literacy practices. California: Sage, 2012.

SQUIRE, Kurt. Mobile media learning: Multiplicities of place. On the Horizon, United Kingdom, v. 17, n. 1, p. 70-80, 2009.

STEINKUEHLER, Constance; COMPTON-LILLY, Catherine; KING, Elizabeth. Reading in the context of online games. International Society of the Learning Sciences, California, v. 1, p. 222-229, 2010.

STRASSER, Katherine; LISSI, María Rosa. Home and instruction effects on emergent literacy in a sample of chilean kindergarten children. Scientific Studies of Reading, Irvine, v. 13, n. 2, p. 175-204, 2009.

STREET, Brian V. Cross-cultural approaches to literacy. Cambridge: New York: Cambridge University Press, 1995. (Cambridge studies in oral and literate culture). 
THIBAUT, Patricia et al. Moving across physical and online spaces: a case study in a blended primary classroom. Learning, Media and Technology, California, v. 40, n. 4, p. 458-479, 2015.

VYGOTSKY, Lev Seminovich. Mind in society: the development of higher psychological processes. Cambridge: Harvard University Press, 1978.

WENGER, Etienne. Communities of practice: learning, meaning and identity. Massachusetts: Cambridge University Press, 1998.

YIN, Robert K. Case study research: design and methods. 4. ed. Thousand Oaks: Sage, 2009.

Recibido en: 28.11 .2018

Aprobado en: 04.06.2019

Patricia Thibaut es Ph.D en Educación de la Universidad de Sydney. Es académica del Instituto de Historia y Ciencias Sociales, Facultad de Humanidades de la Universidad Austral de Chile. Investigadora responsable del Fondecyt $n^{\circ} 3160114$. Sus intereses de investigación abarcan el nexo entre aprendizaje y tecnología en espacios formales e informales.

Margarita Calderón López es PhD en Lingüística de Lancaster University. Actualmente se desempeña como profesora asistente del Departamento de Estudios Pedagógicos de la Universidad de Chile. Sus intereses de investigación abarcan las prácticas de lectura y escritura en la infancia y la enseñanza de lengua indígena la escuela. 